\title{
Beneficiamiento de minerales de caolín
}

\author{
Flor E. Palomar ${ }^{\mathrm{a}}$, Idalia Gómez ${ }^{\mathrm{b}}$ \\ ${ }^{a}$ Universidad Autónoma de Nuevo León, Facultad de Ingeniería Mecánica y Eléctrica \\ ${ }^{b}$ Laboratorio de Materiales I, U.A.N.L. Facultad de Ciencias Químicas \\ *E-mail: maria.gomezd@uanl.edu.mx
}

Recibido 05 de mayo de2014, Aceptado 29 de mayo de 2014.

\section{Resumen}

En el presente trabajo se muestran los resultados de lavado químico y tratamientos térmicos para llevar a cabo procesos de beneficiamiento en tres tipos de caolines, provenientes de la mina Villa de Reyes en el Estado de San Luis Potosí; México. Los tratamientos térmicos llevados a cabo fueron a 1200,1300 y $1350{ }^{\circ} \mathrm{C}$, en rampas de 5 y $20 \circ \mathrm{C} / \mathrm{min}$, en tiempos de estancia de 5 y $30 \mathrm{~min}$. en atmósfera de aire. Las muestras de caolín fueron caracterizadas por difracción de Rayos $\mathrm{X}$ en polvos, espectroscopia por UV-Vis, microscopía óptica y electrónica de barrido. Los análisis por difracción de Rayos X muestran reflexiones características de caolinita, cristobalita y cuarzo, acompañado de cantidades minoritarias de saponita, wollastonita y stellerita. Los análisis por UV-Vis mostraron contenidos de Fe desde 2.24 a $1.80 \%$ en peso. Después del lavado químico y tratamientos térmicos se determinó que el contenido de $\mathrm{Fe}$ en todos los caolines disminuyó, hasta en un $86 \%$ aproximadamente. Obteniendo los mejores resultados a $1350 \circ \mathrm{C}, 20 \circ \mathrm{C} / \mathrm{min}$ y tiempo de estancia $30 \mathrm{~min}$.

Palabras clave: beneficiamiento, caolín, tratamiento térmico.

\section{Abstract}

In this paper the results of chemical cleaning and thermal treatments are shown to perform beneficiation processes in three types of kaolins from the Villa de Reyes mine in the State of San Luis Potosi; Mexico. Heat treatments were performed at 1200,1300 and $1350 \circ \mathrm{C}$, on ramps 5 and $20 \circ \mathrm{C} / \mathrm{min}$, at residence times of 5 and $30 \mathrm{~min}$. in air atmosphere. Kaolin samples were characterized by X-ray diffraction on powders, UV-Vis spectroscopy, optical and scanning electron microscopy. Analyses by X-ray diffraction show characteristic reflections of kaolinite, cristobalite and quartz, together with minor amounts of soapstone, wollastonite and stellerita. The UV-Vis analysis showed Fe content of from 2.24 to $1.80 \mathrm{wt} \%$. After chemical cleaning and heat treatment was determined that the content of Fe in all kaolins decreased by as much as about $86 \%$. Getting the best results at $1350 \circ \mathrm{C}, 20 \circ \mathrm{C} / \mathrm{min}$ and $30 \mathrm{~min}$ residence time.

Keywords: beneficiation, kaolins, heat treatments.

\section{Introducción}

Hoy en día, los materiales cerámicos son una parte natural de edificios y construcciones. El cemento es el material de construcción de hoy y del mañana. El cemento blanco, está constituido por una mezcla de caliza, yeso y caolín [1]. Siendo el caolín uno de los componentes más importantes de este material, éste es una arcilla blanca pura, conocida como arcilla-china. Entre sus importancias industriales se encuentran la cerámica tradicional de pisos, azulejos, baldosas, sanitarios, como en la industria del papel, cerámicas, pinturas, plásticos, caucho, tinta, catalizadores entre otros productos de alta tecnología.

El mineral de caolín más común, y el más importante industrialmente, es la caolinita. La fórmula teórica de la caolinita es $\mathrm{Al}_{2} \mathrm{Si}_{2} \mathrm{O}_{5}(\mathrm{OH})_{4}$ dando una composición química teórica de $46.3 \% \mathrm{SiO}_{2}, 39.8 \%$ $\mathrm{Al}_{2} \mathrm{O}_{3}$ y $13.9 \% \mathrm{H}_{2} \mathrm{O}$. La caolinita se forma fuera del feldespato. [2]. En la tabla 1 se muestran los parámetros estructurales de la caolinita, así como el arreglo estructural.

Tabla 1 Parámetros estructurales de la caolinita.

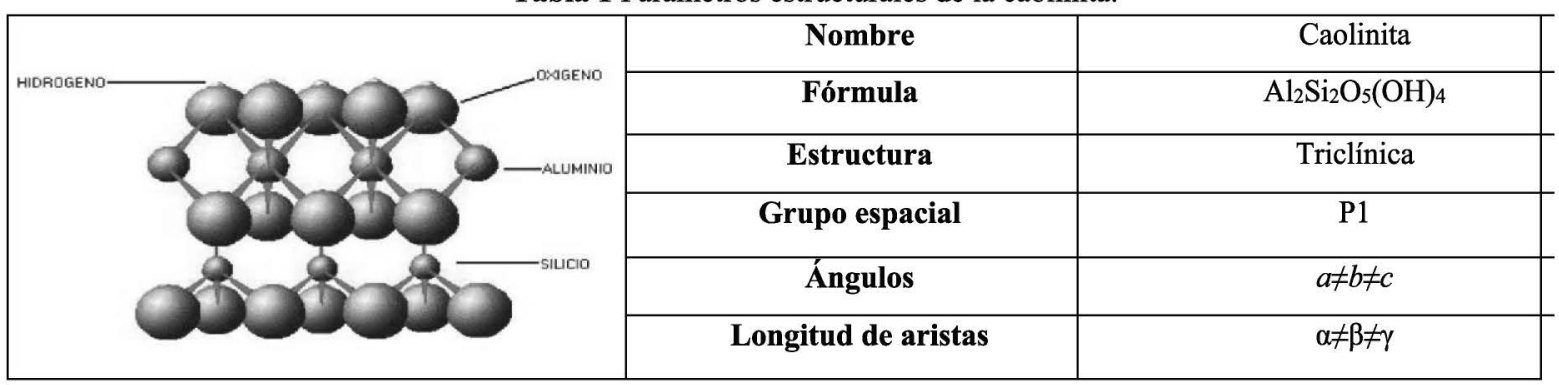


La garantía de obtener caolines con las características de calidad necesarias para la industria, depende en gran parte de la ausencia de elementos cromóforos, tales como $\mathrm{Ti}, \mathrm{C}, \mathrm{Fe}$, etc. Cantidades pequeñas de óxidos de hierro, óxidos de titanio ó sustancias orgánicas disminuyen la blancura de los caolines [3].

$\mathrm{La}$ presencia del mineral cromóforo $\mathrm{Fe}_{2} \mathrm{O}_{3}$ (Hematita) es el que interesa en el presente trabajo. El Fe aparece normalmente en la naturaleza en la forma de ión con tres cargas positivas $\left(\mathrm{Fe}^{3+}\right)$. Casi todos estos minerales son de apariencia oscura, amarillenta o rojiza. Sin embargo, puede presentarse en otro estado de oxidación con dos cargas positivas $\left(\mathrm{Fe}^{2+}\right)$, éstos no son tan oscuros como los minerales que contienen $\mathrm{Fe}^{3+}[2]$. Así, alterando la forma de oxidación del hierro, se tiene una ganancia en el brillo de estas partículas y también del caolín, porque los óxidos férricos se dispersan en el caolín.

Las maneras usuales de conseguir más brillo son agregando un agente de reducción para reducir $\mathrm{Fe}^{3+}$ a $\mathrm{Fe}^{2+}$. La separación magnética es la mejor manera para eliminar los óxidos de titanio. $Y$ en el caso de sustancias orgánicas, se destruyen agregando un agente oxidante, por ejemplo $\mathrm{H}_{2} \mathrm{O}_{2}$ u Ozono $\left(\mathrm{O}_{3}\right)$ [3].

Algunas de las técnicas utilizadas en la actualidad para beneficiar caolines se explican brevemente a continuación:

- Floculación selectiva. Normalmente para las mezclas finas (menores a la malla U.S. 325). Se prepara una mezcla acuosa que contiene los minerales a beneficiar y se acondiciona la misma con la adición de algunos ácidos para obtener una buena floculación. Un ácido comúnmente utilizado es el ácido oleico [4-7].

- Floculación espuma, ésta técnica se utiliza para separar minerales de fosfato con una matriz de sílice, es convencional usar un colector ácido graso y un promotor de sal. Las partículas minerales cubiertas de colector son separadas de la matriz de sílice en la forma de una espuma.

- Otro procedimiento importante en el beneficiamiento de caolines por flotación, especialmente aquellos caolines con impurezas de titanio[8] ha menudo es la ultra flotación . Esta técnica ha sido aplicada por varias décadas para mejorar la calidad de los caolines.

En el presente trabajo se propone estudiar procesos de beneficiamiento a tres tipos de caolines utilizando de manera combinada el lavado químico y tratamientos térmicos, para incidir sobre la blancura de los caolines tratados.

\section{Parte experimental}

Se obtuvieron 3 lotes de caolines de la mina Villa de Reyes en el Estado de San Luis Potosí; México. Los cuales fueron identificados como L64, LKT y L28. Estas muestras fueron caracterizadas por difracción de Rayos X en polvos en un equipo Siemens D5000 utilizando radiación $\mathrm{Cu} \alpha(\lambda=1.5418 \AA)$ la intensidad fue medida en el rango $2^{\circ}$ entre $5^{\circ}$ y $90^{\circ}$ con un paso de $0.05^{\circ}$ para identificar las fases mineralógicas presentes.

El procedimiento experimental seguido fue:

\section{1.- Cribado.}

Con el objetivo de acelerar la disolución, es importante tener el material finamente dividido o pulverizado por lo que se prepararon los lotes de caolines mediante un proceso de homogeneización por el molino cerámico de bolas durante una hora, posteriormente se verificó que al cribar el material a través de la malla $400 \mu \mathrm{m}$ la masa del caolín retenido fuese menor o igual al 3 por ciento de la masa original. 2.- Lavado Químico.

Para poder solubilizar el $\mathrm{Fe}_{2} \mathrm{O}_{3}$ presente en los caolines, se hicieron tratamientos por separado a cada caolín con $\mathrm{HCl}$ concentrado. Los caolines fueron sumergidos en $10 \mathrm{~mL}$ de $\mathrm{HCl}$ y calentando a $80^{\circ} \mathrm{C}$ durante tres horas, observándose una rápida solubilidad. Después de tratarse con $\mathrm{HCl}$, se disolvió en $10 \mathrm{~mL}$ de agua destilada y se pasó por papel filtro, obteniéndose así las muestras necesarias para posteriormente someterlas a tratamientos térmicos.

\section{3.- Tratamiento térmico}

Las muestras una vez lavadas químicamente, fueron sometidas a tratamiento térmico de acuerdo a las cédulas de calentamiento presentadas en la Tabla 2 . Dichos tratamientos se realizaron por medio del uso de platina caliente acoplada a un equipo de microscopía óptica, analizando el proceso mediante un paquete computacional de análisis de imagen. 
Tabla 2. Cédulas de calentamiento.

\begin{tabular}{|c|c|c|}
\hline Temperatura $^{\mathbf{}} \mathbf{C}$ & $\begin{array}{c}\Delta \mathbf{T} / \Delta \mathbf{t} \\
{ }^{\circ} \mathbf{C} / \mathbf{m i n}\end{array}$ & $\begin{array}{c}\text { Tiempo de } \\
\text { estancia (min) }\end{array}$ \\
\hline 1200 & 5 & 5 \\
\hline 1200 & 20 & 5 \\
\hline 1200 & 5 & 30 \\
\hline 1200 & 20 & 30 \\
\hline 1300 & 5 & 5 \\
\hline 1300 & 20 & 5 \\
\hline 1300 & 5 & 30 \\
\hline 1300 & 20 & 30 \\
\hline 1350 & 5 & 5 \\
\hline 1350 & 20 & 5 \\
\hline 1350 & 5 & 30 \\
\hline 1350 & 20 & 30 \\
\hline
\end{tabular}

\section{4.- Determinación de $\mathrm{Fe}$}

Una vez tratadas las muestras térmicamente, se analizó el contenido de Fe por espectrofotometría UVVis, utilizando KSCN para formar un complejo con absorbancia máxima a 480nm.

Se realizó una curva de calibración de 0 a 12 ppm de $\mathrm{Fe}^{3+}$, utilizando $\mathrm{FeCl}_{2}-4 \mathrm{H}_{2} \mathrm{O}$ como patrón, el cual se disolvió en $\mathrm{HCl}$ y se calentó sin llegar a ebullición, durante 20 minutos, con el fin de oxidar el $\mathrm{Fe}^{2+}$ a Fe ${ }^{3+}$. Para la medición de absorbancia, se agregaron $3 \mathrm{~mL}$ de una solución de $\mathrm{KSCN}$ al $2 \%$ y $1 \mathrm{~mL}$ de $\mathrm{HCl}$ concentrado, mientras que para la determinación de $\mathrm{Fe}$

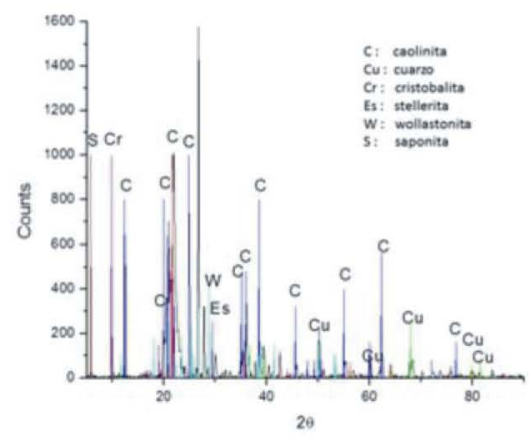

(A) en las muestras, se pesaron $0.0300 \mathrm{~g}$ de cada uno de los caolines y se disolvieron con $10 \mathrm{~mL}$ de $\mathrm{HCl}$, posteriormente se filtró y se agregó la cantidad antes mencionada de KSCN.

El análisis se realizó tanto para las muestras tratadas, como para las muestras que no recibieron tratamiento térmico.

Para la realización de los tratamientos térmicos se utilizó un Microscopio Óptico (Olympus) con platina de calentamiento así como un paquete computacional de análisis de imagen. El análisis de contenido de Fe se realizó en un Espectrofotómetro UV-Vis Perkin Elmer Lambda 12. Las muestras obtenidas se caracterizaron también por microscopía electrónica de barrido (HITACHI S-2600H).

\section{Resultados y discusión}

\section{Difracción de Rayos X}

En la Figura 1 se muestran los espectros de difracción de rayos $\mathrm{X}$ de las muestras de caolín identificadas como L28, L64 y LKT. En éstas se determinó la presencia de fases mineralógicas de cuarzo, cristobalita y wollastonita, las cuales corresponden a polimorfos de la sílice $\left(\mathrm{SiO}_{2}\right)$, así también se identificó a la caolinita y a la saponita, polimorfos del caolín $\left(\mathrm{Al}_{2} \mathrm{Si}_{2} \mathrm{O}_{5}(\mathrm{OH})_{4}\right)$ y a la stellerita que es un aluminosilicato hidratado de calcio, $\mathrm{Ca}_{4}\left(\mathrm{Si}_{28} \mathrm{Al}_{8}\right) \mathrm{O}_{72} \cdot 28 \mathrm{H}_{2} \mathrm{O}$. La muestra LKT denota mayor intensidad en caolinita y el L28 presentó mayor intensidad en cuarzo.

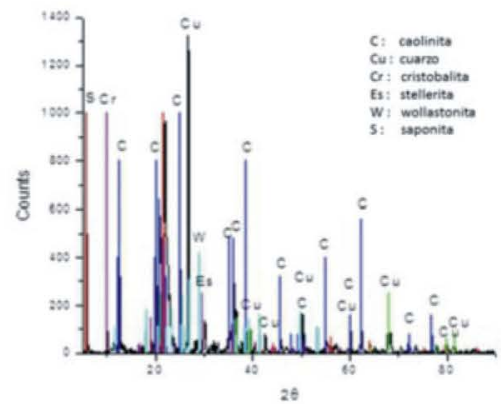

(B)

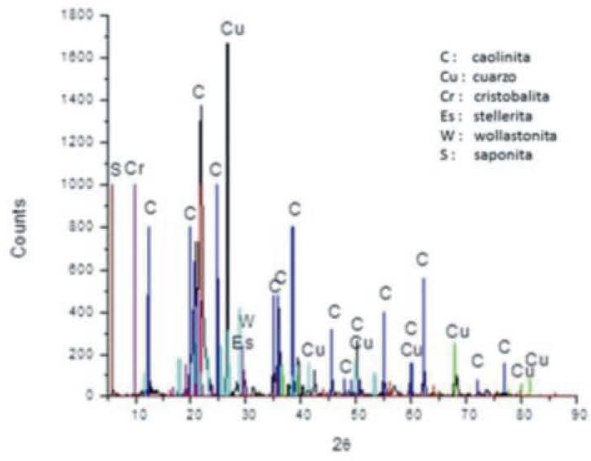

(C)

Figura 1. Espectros de DRX de las muestras L28 (A), L64 (B) y LKT (C). 


\section{Microscopía óptica}

En los 3 caolines se presenta una distribución aleatoria, al igual que la coloración. En general, se observan puntos rojos y/o negros en todas las muestras. Durante todos los tratamientos térmicos se efectuaron procesos similares: algunos puntos rojos toman una coloración negra después del tratamiento térmico, otros puntos desaparecen; y los puntos negros no presentan cambio alguno en la coloración después del tratamiento térmico, otros sólo desaparecen. En la Figura 2 se muestra uno de estos procesos observados. Al término del tratamiento térmico se compara el color del polvo de los caolines y se observa que a $\mathrm{T}=1350^{\circ} \mathrm{C}, \Delta \mathrm{T} / \Delta \mathrm{t}=$ $20^{\circ} \mathrm{C} /$ min y tiempo de estancia de $30 \mathrm{~min}$., presentan mayor blancura.

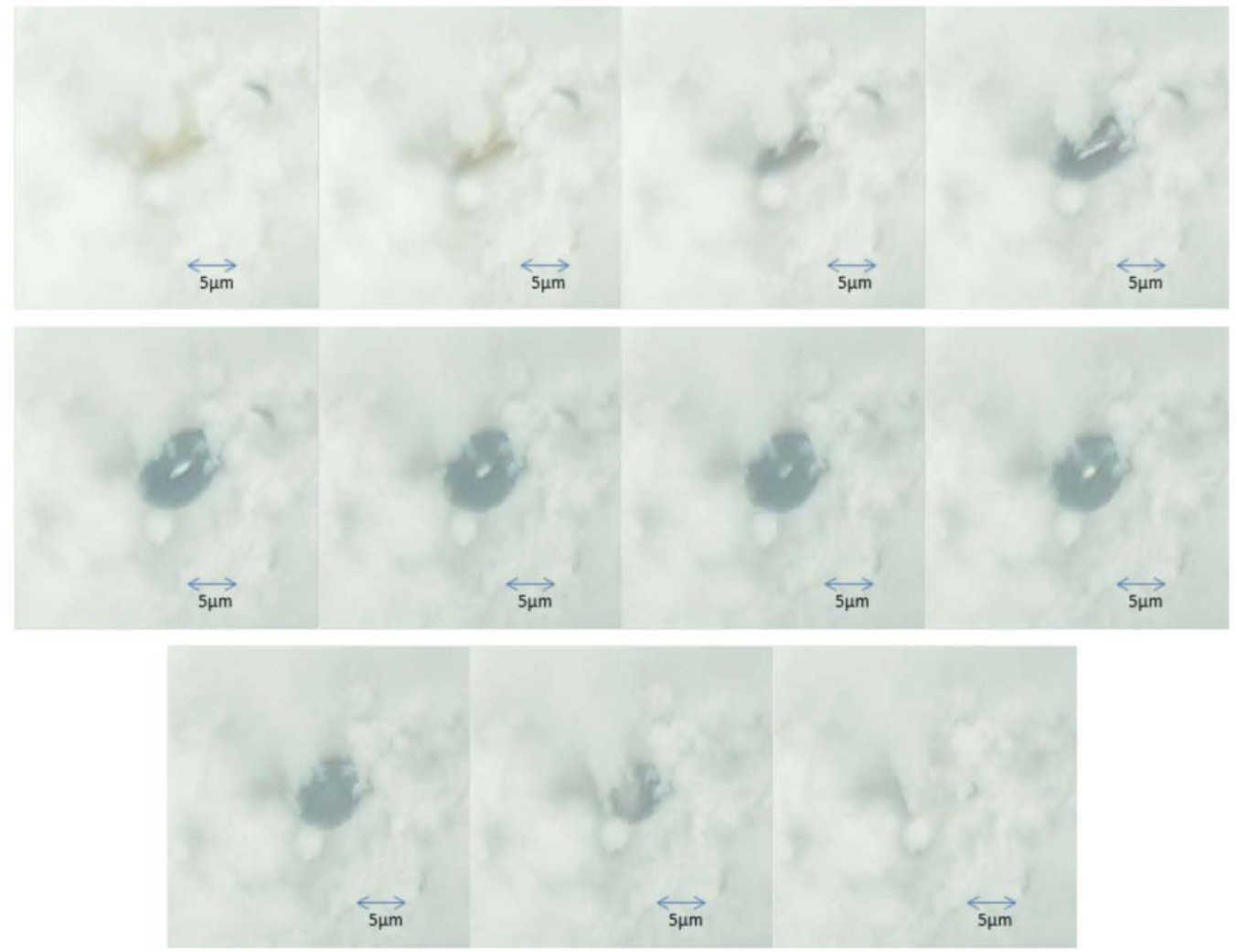

Figura 2. Fenómeno observado en el caolín LKT a $1200 \mathrm{C}$ a $20 \mathrm{C} / \mathrm{min}$ y a los $5 \mathrm{~min}$ de estancia.

\section{Determinación de Fe}

Las concentraciones de Fe obtenidas a partir del método antes descrito, se muestran en la tabla 3 . Donde se puede observar que la concentración de $\mathrm{Fe}$ varía según el lote estudiado, presentando mayor concentración el L28, seguido del L64 y por último el LKT es el que muestra menor concentración. Además la concentración de $\mathrm{Fe}$ disminuye hasta un $86 \%$ aproximadamente, después del tratamiento dado.

Tabla 3. Concentración de $\mathrm{Fe}$ antes y después de los tratamientos térmicos.

\begin{tabular}{|c|c|}
\hline Muestra & $\% \mathrm{Fe}$ \\
\hline L28 original & 2.24 \\
\hline L64 original & 1.85 \\
\hline LKT original & 1.80 \\
\hline L28 tratado & 0.32 \\
\hline L64 tratado & 0.25 \\
\hline LKT tratado & 0.22 \\
\hline
\end{tabular}

\section{Microscopía electrónica de barrido (MEB)}

Se realizaron análisis por MEB a los tres lotes de caolines, con el propósito de estudiar sus características morfológicas así como su micro-estructura. Para lo cual se utilizó la técnica de señal de electrones secundarios.

En el análisis se denota una morfología hexagonal en láminas, característico de la caolinita de acuerdo a lo reportado en literatura[9]. También, por MEB, se pudo observar que durante el proceso de calentamiento se produce un fenómeno de sinterización. En la Figura 3, se aprecia dicho fenómeno térmico en la muestra LKT, al analizar imágenes antes y después del tratamiento térmico por microscopía electrónica de barrido. Dicho fenómeno es debido al contenido de Fe ya que se forma una fase de bajo punto de fusión conocida como Fayalita, cuya fórmula química es $\mathrm{Fe}_{2} \mathrm{SiO}_{4}$ el cual tiene una temperatura de fusión de $1217^{\circ} \mathrm{C}$ [10], lo cual 
provoca el efecto de sinterización en fase líquida aglomerando las partículas de caolín.

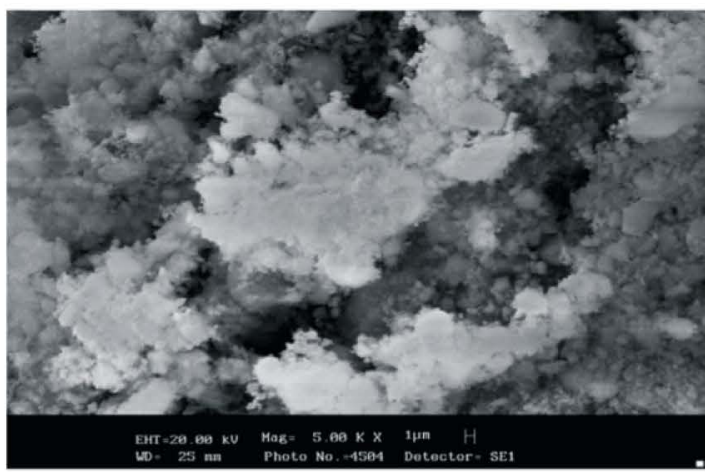

(A)

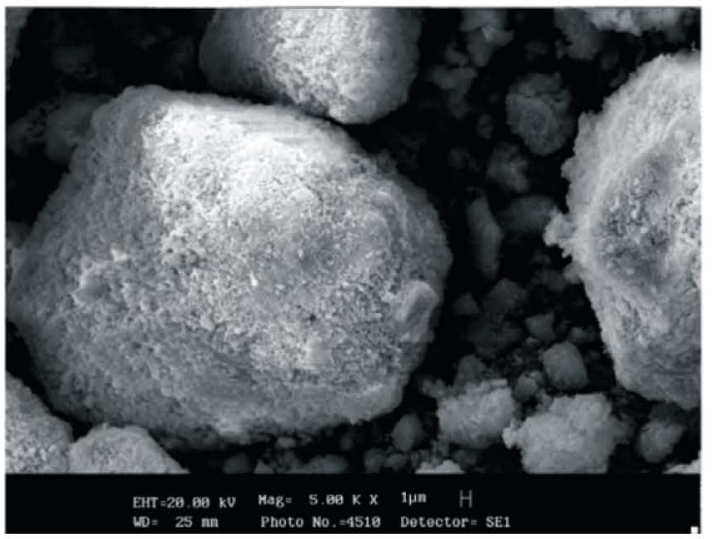

(B)

Figura 3. Micrografías del MEB en el modo de electrones secundarios, muestra de LKT sin tratamiento térmico (A) y con tratamiento (B) a $\mathrm{T}=1350^{\circ} \mathrm{C}, \Delta \mathrm{T} / \Delta \mathrm{t}=20^{\circ} \mathrm{C}$, tiempo de estancia $=30 \mathrm{~min}$. Magnificación de 5000X. Morfología Poligonal y distribución irregular.

\section{Conclusiones}

Los resultados obtenidos en el presente trabajo nos permiten concluir lo siguiente:

- El caolín está constituido principalmente por caolinita, cristobalita y cuarzo, acompañado de cantidades minoritarias de saponita, wollastonita y stellerita.

- El beneficiamiento de caolines se obtuvo en los 3 lotes con todos los tratamientos térmicos, ya que en todos se presentaron cambios con tendencia al blanco.

- El LKT presentó un mayor blanqueamiento a una temperatura de $1350^{\circ} \mathrm{C}$, con una rampa de calentamiento de $20^{\circ} \mathrm{C} / \min$ y un tiempo de estancia de $30 \mathrm{~min}$.

- En todos los minerales de caolín estudiados en el presente trabajo, la morfología de las fases rojizas y oscuras identificadas como impurezas, es puntual.

- La mayoría de las fases de coloración negra que se transformaron se deben a compuestos orgánicos, identificados en mayor proporción en el LKT.

- La transformación de fase de aquellos de color rojizo es un proceso de oxidación del $\mathrm{Fe}, \mathrm{Fe}^{2+}$ $\rightarrow \mathrm{Fe}^{3+}$, es decir, de Hematita cambia a Magnetita.

- El tamaño de partículas identificadas como impurezas es del orden menor a $3 \mu \mathrm{m}$.

- En los caolines tratados térmicamente se da un proceso de sinterización debido a la presencia del Fe.

- El L28 es el de mayor contenido de Fe, seguido del L64 y el LKT.

- El contenido de $\mathrm{Fe}$ en todos los caolines tratados térmicamente disminuye, incluso hasta en un $86 \%$ aproximadamente.

\section{Referencias}

1. Donald R. Askeland, La ciencia e ingeniería de los materiales, Iberoamérica, 1987.

2. Organismo Nacional de Normalización y Certificación de la Construcción y Edificación, S.C., Norma Mexicana NMX-C414-ONNCCE-1999. Industria de la Construcción - Cementos Hidráulicos Especificaciones y Métodos de Prueba.

3. Behl, et al., Metohd for Separating Mixture of Finely Divided Minerals, U.S. Patent : 5,535,890. Julio, 1996.

4. Behl, et al., Colored Titanoferous Coating Pigment Obtained as a Flocculated ByProduct in a Kaolin Purification Process, U.S. Patent : 5,688,315 Noviembre, 1997.

5. Williams, et al., Method for separating Mixture of Finely Divided Minerals, U.S. Patent : 5,603,41. Febrero, 1997.

6. Behl, et al., Colored Titanoferous Coating Pigment Obtaneid as a Floccultaed ByProduct in a Kaolin Purification Process, U.S. Patent : 5,584,394. Diciembre, 1996.

7. Green et al., Use of Removing Impurities from Kaolin Clays, U.S. Patent : 2,990,958 . Mayo, 1998.

8. Norris, et al., Process for Conditioning Kaolin Clays Prior to Removing Impurities, U.S. Patent : 5,685,899. Noviembre, 1997. 
9. J. Guillen, A. S. Bribiesca, y R. Escudero, Caracterización y Evaluación de un depósito de caolín de "Los Azufres" Michoacán, México, para su Uso Industrial, Bol. Soc. Esp. Ceram. Vidr. Vol 51. 6, 329-336, NoviembreDiciembre 2012.

10. F.C. Bacon, Jr., Society for Mining, Metallurgy, and Exploration, Inc., Febrero, 1993. 